\title{
Benjamin Constant et le cercle constitutionnel de 1797 : la modération impossible
}

Benjamin Constant and the Constitutional Circle of 1797: the impossible moderation

Bernard Gainot

\section{(2) OpenEdition}

\section{Journals}

\section{Édition électronique}

URL : https://journals.openedition.org/ahrf/10577

DOI : 10.4000/ahrf.10577

ISSN : 1952-403X

Éditeur :

Armand Colin, Société des études robespierristes

Édition imprimée

Date de publication : 1 septembre 2009

Pagination : 103-118

ISBN : 978-2-200-92559-8

ISSN : 0003-4436

\section{Référence électronique}

Bernard Gainot, «Benjamin Constant et le cercle constitutionnel de 1797 : la modération impossible », Annales historiques de la Révolution française [En ligne], 357 | juillet-septembre 2009, mis en ligne le 01 septembre 2012, consulté le 22 avril 2022. URL : http://journals.openedition.org/ahrf/10577 ; DOI : https://doi.org/10.4000/ahrf.10577 


\title{
BENJAMIN CONSTANT ET LE CERCLE CONSTITUTIONNEL DE 1797 : LA MODÉRATION IMPOSSIBLE
}

Bernard GAINOT

\begin{abstract}
L'histoire des idées politiques présente canoniquement Benjamin Constant comme l'archétype du centre libéral, aussi éloigné des doctrinaires républicains que des partisans de la monarchie absolue, confondus comme défenseurs de la prérogative étatique contre l'autonomie de la société civile. C'est là une réduction téléologique; une étape essentielle de la formation de la pensée de Constant est la période du Directoire. II apparaît alors comme un doctrinaire de la République constitutionnelle, théorisant la primauté de l'esprit public sur l'opinion publique. II faut extraire ce moment fondateur de la pensée politique des habituelles simplifications catégorielles, pour le replacer dans son contexte, celui de la menace de restauration royaliste, et dans ses enjeux théoriques, la définition d'un républicanisme conservateur, qui n'est pas réductible à une posture opportuniste de « juste milieu ».
\end{abstract}

Mots-clés: Directoire, République conservatrice, République constitutionnelle, libéralisme politique, esprit public, opinion publique.

Analysant le coup d'État du 18 fructidor an V avec un recul de vingt ans ${ }^{1}$, Germaine de Staël y voit un moment inaugural de l' "état d'exception » dans lequel la France restera plongée jusqu'en 1814. Le

(1) Germaine DE STAEL, Considérations sur la Révolution française, présentation de Jacques Godechot, Paris, Tallandier, 2000 ( $1^{\text {re }}$ édition, 1983). 
chapitre XXIV des Considérations sur la Révolution française s'intitule : «De l'introduction du gouvernement militaire en France, par la journée du 18 fructidor ». Tout est dit ; Fructidor préfigure Brumaire et ce qui va suivre. La République constitutionnelle, née en 1795, a reçu un coup fatal, après deux années d'existence ${ }^{2}$. Cette version a été reprise dans un ouvrage récent, qui souligne tout à la fois la volonté des thermidoriens d'établir un authentique régime libéral en 1795, puis la dérive autoritaire d'un «État sécuritaire » qui trouve sa réalisation dans une militarisation progressive de la République, dont Fructidor, aussi bien que Brumaire, ne sont que des étapes vers un dispositif qui se déploie pleinement à partir de 1802 . La situation démontrerait l'impossibilité de stabiliser un centre libéral, sous la pression d'une montée aux extrêmes, que seul un « extrémisme du centre » parviendrait à juguler ${ }^{3}$. A contrario, Isser Woloch montre lui aussi, l'importance de la journée du 18 fructidor dans la construction de ce qu'il appelle la « dictature » napoléonienne, mais de façon réactive ; le régime issu de Brumaire cherche avant tout à effacer la mémoire de cette inflexion « républicaine » du Directoire. Pour lui, le centre est par nature introuvable, et deux logiques n'ont cessé de s'affronter jusqu'à ce que la logique républicaine sombre durablement face à la logique du pouvoir personnel ${ }^{4}$.

Le sens de la journée, tel que le donne Germaine de Staël a posteriori, est à rechercher à l'aune du décalage entre les institutions et les héritages. Selon elle, la Constitution de 1795 est une bonne constitution, supérieure à celle de 1791. Un éloge appuyé de trois de ses concepteurs est proposé : Boissy d'Anglas, Daunou et Lanjuinais. Deux réserves de taille nuancent cependant ce jugement positif : «Ce pouvoir exécutif n'avait point encore l'autorité nécessaire pour maintenir l'ordre; il lui manquait plusieurs prérogatives indispensables, et dont la privation amena, comme on le verra dans la suite, des convulsions destructives $»^{5}$. Et une république fédérative aurait été préférable à la république une et indivisible, « il aurait fallu peut-être sacrifier Paris à la France », mais ce

(2) Sur les diverses interprétations du 18 fructidor: Albert Meynier, Les coups d'État du Directoire, tome 2, Paris, PUF, 1928 ; Georges LefebVRe, La France sous le Directoire, présentation de Jean-René Suratteau, Paris, Éditions sociales, 1977 ; Howard Brown, Ending the Revolution; violence, justice and repression, from the Terror to Napoleon, University of Virginia Press, 2006.

(3) Pierre SERna, la République des girouettes. 1789-1815 et au-delà. Une anomalie politique : la France de l'extrême centre, Seyssel, Champ Vallon, 2005.

(4) Isser Woloch, Napoléon and its collaborators. The making of a dictatorship, New-York, Norton, 2001.

(5) Considérations, op. cit., p. 319. 
n'était pas dans le caractère national. Les mesures révolutionnaires contrebalancent encore l'ordre constitutionnel. Le Directoire tire essentiellement sa force de ce qu'il constitue « une faction révolutionnaire », en l'absence de moyens légaux que sont le droit de dissolution, et le veto législatif. « La majorité du Directoire, Rewbell, Barras, et La Révellière, hésitèrent quelque temps entre deux auxiliaires dont ils pouvaient également disposer; le parti jacobin, et l'armée $»^{6}$. Ils se tournèrent finalement vers l'armée, parce qu'ils redoutaient par-dessus tout les jacobins. Mais on retiendra de ce parallèle que l'armée est vue comme substitut de la force populaire, et le gouvernement militaire comme un nouvel avatar du gouvernement révolutionnaire.

« C'est alors que l'on vit s'établir entre la force populaire et la force militaire une alliance qui rendit bientôt celle-ci maîtresse de l'autre. Les guerriers français, si admirables dans la résistance qu'ils opposaient aux puissances coalisées, se sont faits, pour ainsi dire, les janissaires de la liberté chez eux et, s'immisçant dans les affaires intérieures de la France, ils ont disposé de l'autorité civile, et se sont chargés d'opérer les diverses révolutions dont nous avons été les témoins ${ }^{7}$.

Par un retour en arrière, $\mathrm{M}^{\mathrm{me}}$ de Staël expose que l'esprit révolutionnaire est au cœur même de l'acte de naissance du Directoire, avec le décret des deux tiers, qui provoqua la journée du 13 vendémiaire, qui provoqua ensuite le décret du 2 brumaire ; ainsi l'état d'exception est au cœur de l'ordre constitutionnel.

«Le décret d'exclusion du 2 brumaire faisait une classe de proscrits dans l'État, ce qui, certes, ne vaut pas mieux qu'une classe de privilégiés, et n'est pas moins contraire à l'égalité devant la loi »".

Ce faisant, Germaine de Staël n'en exonère pas pour autant le «parti royaliste », c'est-à-dire ce regroupement de la droite, improprement appelée «modérée », réunie dans le Club de Clichy, au sein duquel elle a de nombreuses relations. Elle analyse ce parti, en soulignant qu'il invoque dans son combat les principes républicains ; liberté de la presse, liberté des suffrages, droit à l'insurrection. Il contraint ses adversaires républicains à se placer sur la défensive, et à désavouer ses propres prin-

(6) Ibid., p. 330-331.

(7) Ibid., p. 319-320.

(8) Ibid., p. 321. 
cipes en invoquant les circonstances, comme l'avait fait le gouvernement révolutionnaire à l'époque de la Terreur. C'est en quelque sorte une lutte à front renversé. Elle dénonce la précipitation et les menaces des clichyens dès qu'ils eurent remporté les élections de l'an V.

« Au moment où le second tiers des chambres fut renouvelé par l'élection de 1797, l'esprit public devint une seconde fois impatient d'écarter les conventionnels des affaires; mais une seconde fois aussi, au lieu d'attendre une année pendant laquelle la majorité du directoire devait changer, et le dernier tiers des chambres se renouveler, la vivacité française porta les ennemis du gouvernement à vouloir le renverser sans nul délai... On vit s'agiter de toutes parts un esprit de réaction intolérable $»^{9}$.

Si on comprend bien, $\mathrm{M}^{\mathrm{me}}$ de Staël semble d'abord considérer avec impartialité la droite, dont elle relate avec intérêt la tactique, pour s'en détourner lorsque, saisie par un processus de radicalisation, elle met en péril la stabilité constitutionnelle, et précipite le pays vers la contre-révolution, donc vers la guerre civile (esprit de vengeance, assassinats, mesures vexatoires tout particulièrement dans le domaine religieux). Cette analyse pose plusieurs problèmes, que Madame de Staël aborde de façon subjective, en faisant part de sa position personnelle de façon indirecte. Le reproche de lecture rétrospective (comprendre Fructidor par l'épilogue de Brumaire, y voir la marque inéluctable du gouvernement militaire) est bien présent à son esprit. Aussi va-t-elle intercaler dans le récit un chapitre consacré à l'ouvrage publié par Necker à la fin de l'année 1796 sur l'histoire de la Révolution française. Ce chapitre s'intitule de façon significative : «Deux prédictions singulières tirées de l'Histoire de la Révolution par M. Necker ». L'une de ces prédictions porte sur l'avènement du gouvernement militaire, conséquence de l'abaissement du pouvoir exécutif. Ainsi elle peut se prévaloir de la proximité du grand homme pour écarter le reproche de réécriture téléologique de l'histoire, mais aussi pour répondre aux attaques qui mettent en doute sa distance à l'égard du coup d'État.

« Il en coûte de parler de soi, dans une époque surtout où les récits les plus importants commandent seuls l'attention des lecteurs. Néanmoins, je ne puis me refuser à repousser une inculpation qui me blesse. Les journaux

(9) Ibid., p. 329-330. 
chargés, en 1797, d'insulter tous les amis de la liberté, ont prétendu que, voulant la république, j'approuvais la journée du 18 fructidor $»^{10}$.

Elle borne son intervention active à un rôle d'intermédiaire entre Barras et Talleyrand, afin que ce dernier soit nommé au ministère des Relations extérieures, en juillet 1797. Elle prend bien soin de découpler ce changement ministériel, de la « révolution du 18 fructidor », qui « doit toujours faire horreur, et par les principes tyranniques dont elle partait, et par les suites affreuses qui en ont été la conséquence nécessaire ». Effectivement, la valse des ministres en juillet fut l'ultime tentative de compromis entre le triumvirat directorial (Rewbell, La Revellière et Barras) et le Conseil des Cinq-Cents dominé par les clichyens. L'arrivée de Talleyrand et de Pleville Le Pelley devait équilibrer celle de Hoche et de François de Neufchâteau, le départ de Truguet et de Charles Delacroix compenser celui de Cochon de Lapparent et de Petiet. Énorme marchandage qui s'apparenta bientôt à une « journée des dupes » favorable au Directoire.

Le sentiment de Madame de Staël sur le 18 fructidor fut-il bien empreint de cette horreur détachée dont elle nous fait part vingt ans plus tard ? Bien qu'elle ait cherché à en corriger les limites, cette interprétation est une lecture rétrospective. Pour approcher au plus près de l'événement, nous allons rendre compte des opinions de ceux dont elle était alors très proche, et qui furent non seulement des témoins directs, mais aussi des acteurs engagés, regroupés dans le Cercle constitutionnel de la rue de Lille. Puis nous nous interrogerons sur la réalité d'un centre, que nous inclinons à juger tout aussi introuvable en 1797 qu'il pouvait l'être en $1790^{11}$.

Le $\mathrm{n}^{\circ} 28$ de La décade philosophique du 10 messidor an $\mathrm{V}$ (28 juin 1797) nous fait part de la formation du Cercle constitutionnel de la rue de Lille, dont les principes sont rapportés avec approbation : « Opposer à la réaction contre les principes libéraux, qui menace de devenir une réaction contre la Constitution, une barrière d'autant plus forte qu'elle serait constitutionnelle ». La réaction ainsi visée est celle qui s'organise autour $\mathrm{du}$ Club de Clichy. La première réunion du Cercle constitutionnel a rassemblé environ cinq cents personnes, dont Jourdan, Kléber, Menou, Cailhava, Duveyrier, Joseph Eschassériaux, Lacépède, Lecouteulx de

(10) Ibid., p. 333.

(11) Robert Griffiths, Le centre perdu. Malouet et les monarchiens dans la Révolution française, Grenoble, Presses Universitaires de Grenoble, 1988. 
Canteleu, Talleyrand. Certes, $\mathrm{M}^{\mathrm{me}}$ de Staël n'est pas mentionnée, mais son nom est cité dans d'autres sources et, de toutes façons, on reconnaît dans ces figures de proue plusieurs de ses proches, à tel point que l'on peut avancer sans se tromper qu'elle en partageait les principes. Quant à son compagnon Benjamin Constant, il en deviendra bien vite au fil des semaines la figure la plus représentative.

Autre point à relever dans le même numéro de La décade, une critique acerbe, sous la plume de Ginguené, du livre de Necker, dont Germaine fait l'apologie dans les Considérations. Cette critique a occupé plusieurs numéros de La décade, depuis le $n^{\circ} 23$ (20 floréal an V). Le jugement, nuancé sur la forme, est sans appel sur le fond : Necker est un apostat. Il est animé par le ressentiment, né de la confiscation de ses biens par suite de ses positions monarchiques, et il n'est bon que comme mémorialiste, lorsqu'il décrit les événements auxquels il a personnellement participé. Mais, depuis 1791, il est éloigné de ce qu'il décrit, et son analyse est entièrement faussée par l'aigreur du ton. Il est devenu étranger à la France républicaine.

Pour la proximité de Benjamin Constant et de Germaine de Staël à cette époque, les faits sont suffisamment connus pour que nous n'ayons pas à reprendre le dossier ${ }^{12}$. Aussi bien les détracteurs du couple, comme Henri Guillemin, que les biographes qui voulurent rectifier ce qu'ils estimaient être un parti-pris systématique de dénigrement, comme Béatrice Jasinski, conviennent que la grande brochure De la force du gouvernement actuel, et de la nécessité de s'y rallier, fut écrite à quatre mains lors du séjour du couple à Coppet, au cours de l'hiver 1796, et c'est ce qui nous importe. Mais un infléchissement allait se produire un an plus tard, avec la brochure qui porte plus nettement la marque du seul Constant, Des réactions politiques. Les deux auteurs partent d'un positionnement interne à la droite ; c'est en priorité aux « modérés » qu'ils s'adressent quand ils leur demandent de se rallier à la République, comme gouvernement établi. Il s'agit d'un dialogue prioritaire avec des publicistes de droite comme Suard ou Charles Lacretelle. Le fait de savoir si l'une ( $\mathrm{M}^{\mathrm{me}}$ de Staël) voulait davantage les ménager que l'autre (Constant) est secondaire, somme toute, au regard de l'argumentation de fond. Henri Guillemin avance de façon abrupte, et réductrice, qu'il s'agissait de rallier les possé-

(12) Béatrice JAsINSKI, L'engagement de Benjamin Constant, amour et politique (17941796), Paris, Minard, 1971. 
dants au nouveau régime ${ }^{13}$. Ce qui importe, c'est qu'il s'agit bien d'une controverse interne à la droite, aux républicains par raison.

Face aux critiques des clichyens, bien exprimées dans la brochure de Lezay-Marnézia, Constant infléchit sa position vers un républicanisme plus affirmé, qui s'affiche dans la brochure intitulée Des réactions politiques. Tandis que Madame de Staël reste sur la position initiale, Constant s'engage beaucoup plus dans l'action, et notamment au Cercle constitutionnel. Une dizaine de jours après le 18 fructidor, il prononce le grand discours devant ce cercle, dans lequel il traduit en formules militantes les positions théoriques affichées dans ses précédentes brochures, tout en manifestant, clairement cette fois, son opposition à la position exprimée par Lezay-Marnezia.

C'est ce discours que nous allons maintenant analyser d'un peu plus près ${ }^{14}$. Le ton général est celui d'un discours-programme, et non celui d'un simple discours d'adhésion à l'événement. Le ton est très différent de celui des adresses qui parviennent de tous les points du territoire dans les mois qui suivent pour féliciter le Directoire d'avoir osé agir. Constant n'est pas un porte-parole, c'est un particulier qui fait part de sa démarche intellectuelle, de son parcours politique personnel en évolution, aux frontières de la sphère publique et de la sphère privée. Il faut donc prendre ce discours comme l'expression d'une posture de ralliement, qui, par-delà le public effectif du Cercle constitutionnel, s'adresse en fait à d'autres interlocuteurs.

Et d'abord à ses amis de la « jeunesse dorée », y compris $\mathrm{M}^{\mathrm{me}}$ de Staël, mais encore Lacretelle ou Suard, qui ont condamné le décret des deux tiers, et soutenu les sectionnaires parisiens du 13 vendémiaire :

«On a pensé que pour conclure une paix solide avec l'aristocratie, il fallait la placer bien commodément dans la République»Et, toujours dans le même esprit : «Les préjugés, l'orgueil, la cupidité, la vengeance, la superstition, toutes les passions ignobles ou furieuses, se sont ralliées autour de l'idée d'un Roi. On a cru longtemps qu'en donnant, sous la République, à toutes ces passions hostiles ou corruptrices, toutes les jouissances qu'elles pourraient exiger, on parviendrait à leur faire aimer le gouvernement républicain $»^{15}$.

(13) Henri Gullemin, Benjamin Constant, muscadin. 1795-1799, Paris, Gallimard, 1958.

(14) Discours prononcé au Cercle constitutionnel pour la plantation de l'arbre de la Liberté, le 30 fructidor an V, par Benjamin Constant, Paris, BNF, Lb ${ }^{40} 2360$.

(15) Ibid., p. 7. 
Qui donc est ce « on », résolument placé sous le signe des illusions perdues ? L'argumentaire revient au dialogue conflictuel noué avec LezayMarnezia, et tourne autour de la notion de «majorité nationale ». Cette «majorité nationale », c'est l'opinion publique, telle que définie justement par les publicistes de droite, auxquels Constant s'est d'abord agrégé. Ce sont les idées dominantes après Thermidor, véhiculées dans les salons parisiens, et hors de ceux-ci par la jeunesse dorée, c'est la guerre des symboles, les rixes dans la rue, les incidents dans les lieux de spectacle. C'est dans ces espaces délimités par les cafés, les promenades et les théâtres, que se fait l'opinion publique. Il reste à la transformer en bulletins de vote, et on aura la «majorité nationale». Dans les Réactions politiques, Benjamin Constant avait très bien saisi à quel point toutes ces manifestations réactionnaires étaient portées par la nostalgie :

«Ces réactions qui, de révolutions désastreuses, font encore des révolutions inutiles, naissent de la tendance de l'esprit humain à englober dans ses regrets tout ce qui entourait ce qu'il regrette. Ainsi que dans nos souvenirs de l'enfance, ou d'un temps heureux qui n'est plus, les objets indifférents se mêlent à ce qui nous était le plus cher, et le charme du passé s'attache à tous les détails, l'homme qui, dans le bouleversement général, a vu s'écrouler l'édifice de son bonheur individuel, croit ne pouvoir le relever qu'en rétablissant tout ce qui partagea sa chute; les inconvénients même et les abus lui deviennent précieux, parce qu'ils lui paraissent dans le lointain, liés intimement aux avantages dont il déplore la perte $»^{16}$.

Mais il ne reprend plus dans son discours au Cercle constitutionnel, cette tonalité mélancolique qui est aussi celle des amours perdus, pour consacrer sa rupture définitive avec une façon de penser qui fut la sienne. « [...] Ne nous perdons plus en conciliation trompeuses, ne nous consumons plus en sacrifices gratuits $\gg^{17}$. Tout d'abord, parce que la réaction, c'est aussi et d'abord l'esprit de vengeance, et cet esprit de vengeance est au cœur d'un nouveau radicalisme, qui débouche sur une nouvelle révolution, elle-même porteuse de la guerre civile. L'esprit révolutionnaire a changé de sens, mais c'est toujours l'esprit révolutionnaire. Il était porté par l'enthousiasme au début de la décennie, il est maintenant porté par le ressentiment et la nostalgie.

(16) Benjamin Constant, Des réactions politiques; Des effets de la Terreur, présentation de Philippe Raynaud, Paris, Flammarion, 1988.

(17) Discours au Cercle Constitutionnel, op. cit., p. 10. 
Cette inversion de sens que Lezay-Marnezia a captée sous le nom de «majorité nationale », Constant l'invoque sous le nom de la mode. Citons ce long passage, car c'est le pivot de son discours : " Ce que la classe aristocratique doit perdre en pouvoir, il ne faut pas qu'elle puisse le reconquérir en influence indirecte [...] implacable sans moralité ${ }^{18} \gg$. C'est la consécration d'une rupture qui s'affiche ici ; pour reprendre le titre de l'ouvrage de Henri Guillemin, si Benjamin Constant a été muscadin, il prend ici une distance absolue par rapport à cette posture. Et il se tourne vers le Directoire triomphant. Le rôle d'un gouvernement républicain, ce n'est pas de suivre l'opinion publique, ce n'est pas de suivre la mode. Il aborde la partie proprement politique, et il reprend pour cela un argumentaire déjà abordé dans De la force du gouvernement actuel : le gouvernement républicain ne tolère pas l'indifférence à l'égard des formes :

« Repoussons, Citoyens, ce système d'indifférence que l'on décore du nom d'impartialité [...] Pour faire marcher la liberté, il faut être partial pour la liberté ; et lorsqu'on la voit d'une part, et ses ennemis de l'autre, il ne faut pas se faire un mérité imbécile de savoir rester au milieu ».

Il entre dans la question de l'administration. La droite s'appuyait sur le parti dit des « honnêtes gens », qui prétendait occuper les fonctions publiques, pour faire coïncider ses intérêts avec l'ordre constitutionnel, en laissant dépérir les institutions républicaines. Le gouvernement républicain n'est pas le gouvernement des intérêts :

« Ceux qui ne croient pas aux droits du peuple, doivent être déshérités de ces droits. Qu'ils vivent paisibles, mais sans puissance, assurés de leurs biens, mais sans éclat, garantis dans leur insignifiance, mais hors d'état d'en sortir et, frappés d'une nullité salutaire, qu'ils ne puissent jamais, s'introduisant dans les fonctions publiques, attiédir le saint enthousiasme qui nous anime [...]. Il ne faut pas que, sur toute l'étendue de la république, il se trouve, dans une fonction quelconque, depuis l'administrateur municipal de la plus petite commune jusqu'aux dépositaires suprêmes de l'autorité exécutive, depuis le commis le plus subalterne du bureau le plus obscur, jusqu'au ministre chargé de la gestion la plus importante, un seul homme qui ne soit solidaire de la liberté républicaine, qui n'ait contracté envers elle d'indissolubles engagements, qui ne porte en son âme la confiance de sa force, la certitude de sa durée, et l'abandon du plus entier dévouement pour elle ${ }^{19}$.

(18) Ibid., p. 14-16.

(19) Ibid., p. 13. 
Certes, prudemment, Constant ne reprend pas la métaphore militaire développée dans De la force du gouvernement actuel :

«Avez-vous vu quelquefois, dans une bataille, une phalange épaisse de soldats, s'avançant serrés l'un contre l'autre, de manière à ce que la vue ne perce pas au-delà du premier rang ? Ils ne paraissent vouloir combattre qu'avec les armes qu'ils ont en main: on ne se prépare qu'à repousser le choc, dont ils menacent. Tout à coup, ils s'arrêtent, font un mouvement subit, s'entrouvrent : une artillerie formidable se fait voir, et vomit sur l'ennemi pâlissant l'épouvante et la mort.

Les terroristes sont cette artillerie du gouvernement, toujours cachée, mais toujours redoutable, et qui, toutes les fois qu'il sera forcé de l'employer, réduira en poudre ses adversaires $\gg{ }^{20}$.

Cette métaphore lui avait valu de très vifs reproches, et certainement la désapprobation de $\mathrm{M}^{\text {me }}$ de Staël. Et, pourtant, elle illustrait remarquablement la pratique administrative du Directoire naissant : destituer des administrateurs tièdes ou suspects de sympathies royalistes, les remplacer par des patriotes qui avaient été victimes de la réaction. Pour défendre la République, prolonger l'état d'exception dans l'ordre constitutionnel, en définissant les contours d'un «ennemi intérieur », avec lequel tout compromis est impossible. Le Directoire revient à cette pratique après Fructidor. Constant la justifie : « la constitution lui confie [au Directoire] le pouvoir salutaire des destitutions $»$.

Puisque les formes ne sont pas neutres, il reste à les caractériser ; les attributs du régime sont la « prévoyance » (prévenir la radicalisation de la droite), la « sagesse » (le salut public sans la peine de mort), et la «force morale des institutions ». Tous ces attributs se résument en un seul : la puissance. Cette ligne n'est certes pas nouvelle. Elle a déjà été mise en avant en deux circonstances cruciales de la période thermidorienne. Lors des journées de prairial an III, plusieurs journalistes du courant démocratique avaient condamné la radicalisation populaire comme un prétexte pour accentuer la réaction royaliste. Un réflexe identique conduisit ces mêmes journalistes à accueillir froidement les premiers numéros du Tribun du peuple qui attaquaient le nouveau régime constitutionnel. Le Journal des patriotes de 89 dénonce ainsi les risques que fait courir à l'ensemble du courant républicain les diatribes du Tribun : « Si

(20) De la force du gouvernement actuel de la France et de la nécessité de s'y rallier (microfiche Pergamon, 6.3/205). 
j'étais royaliste, je ferais en sorte que les chouans vinssent dire à la tribune ; les terroristes relèvent la tête [...] les voilà qui provoquent l'anéantissement de la Constitution que vous avez décrétée $»^{21}$. Le danger principal est identifié comme venant de la droite, il faut serrer les rangs (encore une métaphore militaire) derrière le régime constitutionnel, parce qu'il est le conservatoire des acquis républicains. Il n'y a plus de force co-active, parce qu'on sait désormais que le peuple peut errer, qu'il n'a pas de principes intangibles. C'est là une différence notable avec le premier républicanisme, celui d'avant la Terreur. Désormais, toute l'énergie est concentrée dans le gouvernement, c'est là qu'est « la puissance ».

Cet argumentaire était déjà celui de Marie-Joseph Chénier en messidor an III (juin 1795) à la tribune de la Convention lorsqu'il dénonçait les crimes royalistes de la Terreur blanche :

"C'était, si l'on eût cru Marius et Sylla, pour sauver la République Romaine, qu'ils dressaient les tables sanglantes de leurs proscriptions ; c'était pour sauver la République française que Robespierre et ses nombreux complices, couvraient la France d'échafauds [...] c'était pour sauver la liberté que des scélérats égorgeaient, le 2 septembre, dans des prisons de Paris ; c'était encore pour sauver la liberté que les mêmes hommes assiégeaient la Convention nationale le 31 mai. [Le] principe d'humanité [, qui fut invoqué pendant la Terreur, est maintenant invoqué par les royalistes et autres] égorgeurs royaux [...]. La mode a changé pour les mots, mais la marche des factions reste et restera la même ; c'est donc au nom de l'Humanité qu'on égorge maintenant à Lyon et dans quelques cités du midi de la France ; ce serait au nom de la Convention nationale que la Convention nationale serait égorgée $[\ldots] »^{22}$

La seule façon de rétablir l'ordre constitutionnel serait donc de violer la Constitution en destituant des administrateurs élus, de mettre en place un régime d'exception, pour mieux combattre ceux qui invoquent la légitime défense et le salut commun, de restreindre les libertés individuelles pour mieux garantir les libertés publiques. Cette ligne de défense républicaine, qui est au fondement de la République « conservatrice », c'est donc celle invoquée par Constant dans son discours.

(21) Citation dans Laura MAson, «Après la conjuration, le Directoire, la presse, et l'affaire des Égaux » dans Annales historiques de la Révolution française, $n^{\circ} 354,2008$, p. 83 ; et Bernard GAINOT, «Babeuf à travers la presse démocratique et républicaine en l'an IV », dans Babeuf et les babouvistes en leur temps, Saint-Quentin, les Amis de Gracchus Babeuf, 2000, p. 101.

(22) Le Moniteur, $\mathrm{n}^{\circ}$ du 10 messidor an III. (28 juin 1795). 
Il reste à inscrire le 18 fructidor dans la ligne de l'histoire, dans la suite du 14 juillet et du 10 août, notamment. Mais surtout à montrer que l'intervention de la force n'est pas contradictoire avec la République constitutionnelle, qui reprend son cours :

« Aussi voyons-nous les bannières de la Constitution, un instant abaissées pour l'intérêt général, se relevant aussitôt, et consacrées doublement par le respect unanime, par l'implicite résignation de toutes les ambitions particulières. Ces trois directeurs, qui avaient sauvé la Patrie, ont appelé deux nouveaux collègues au partage de leur puissance. Ils ont courbé toute considération personnelle devant la constitution, eux qui avaient fait plier la Constitution devant le salut de la république; et du nombre des candidats à ce rang suprême, s'est retiré le général de cette nuit mémorable, parce qu'il s'était éloigné de quelques jours de l'âge prescrit par la loi ».

Car, et c'est le dernier point, Constant réfute l'idée du gouvernement militaire. Tout d'abord, en établissant le parallèle qu'il reprendra deux ans plus tard, avec la Révolution anglaise. La république a pu se sauver sans faire appel au Protectorat, sans susciter un nouveau Cromwell. Mais, préfigurant en quelque sorte le plan que développera $\mathrm{M}^{\text {me }}$ de Staël l'année suivante, il avance l'idée d'une intégration des généraux dans le Corps législatif : " Alors, nous n'aurons plus à craindre le gouvernement militaire, puisque l'éclat militaire ne fera que rehausser le gouvernement civil $»^{23}$.

Il faut maintenant replacer cet engagement de Constant (seul engagement positif de celui-ci, avec la rédaction de l'Acte additionnel en 1815) dans son environnement idéologique. Dans les mois qui suivent le coup d'État de Fructidor, la sociabilité républicaine connaît un formidable renouveau, avec la multiplication dans toute la France des cercles constitutionnels. Mais l'orientation de ces sociétés politiques est assez éloignée du programme inspiré par Constant. Dans la capitale, j'ai pu dénombrer dix-sept cercles constitutionnels ${ }^{24}$; il y en a au moins un par arrondissement. Certains sont très radicaux, et, significativement, ne reprennent pas l'intitulé «cercle constitutionnel», mais s'appellent plus volontiers « réunion politique ». On en compte cinq dans le seul faubourg SaintAntoine, qui font de la surenchère dans le radicalisme démocratique. Les deux cercles constitutionnels les plus fréquentés sont toutefois ceux du

(23) Discours, op. cit., p. 22.

(24) Atlas historique de la Révolution française, tome 11 (Émile Ducoudray, Raymonde Monnier, Daniel Roche, Alexandra Laclau, dir.) Paris, Editions de 1'EHESS, 2000, p. 63. 
dixième arrondissement ; le premier est celui de l'Hôtel de Montmorency, à la tribune duquel nous venons de voir Benjamin Constant prononcer son discours. À la fin du mois de février 1798, il transfère le lieu de ses séances de la rue de Lille à l'ancien Palais-Royal ${ }^{25}$.Lenoir-Laroche fait l'éloge de ses activités, et rappelle les circonstances de sa naissance ; un acte décisif dans la lutte contre la «faction conspiratrice » des clichyens :

"Quelques patriotes, aussi courageux qu'éclairés n'eurent pas plus tôt formé le projet de se réunir pour lutter contre cette affreuse perversité, qu'aussitôt tous les amis de la liberté accoururent en foule. Ce fut, dans les départements comme à Paris, le signal de leur réveil; cette phalange d'amis et de défenseurs des principes républicains parut $s i$ formidable aux royalistes, qu'ils ne se crurent un instant rassurés qu'en obtenant la clôture de tous les cercles, par le sacrifice même de celui de Clichy $[\ldots]$ Cependant, les cercles avaient produit leur effet. Ils avaient révélé au gouvernement la puissance de l'opinion, et le nombre des appuis qui entouraient la constitution pour la défendre. Le 18 fructidor en fut le résultat ».

Dans cette approche rétrospective, le discours de Constant a fait date :

« On se souvient des discours qui furent prononcés lors de la cérémonie de la plantation de l'arbre de la liberté, et de l'impression que produisit entre autres celui d'un de ses membres, Benjamin Constant, qui exprima avec tant d'énergie les principes que les vrais républicains ne doivent jamais perdre de vue, $s^{\prime}$ ils veulent conserver l'organisation sociale qu'ils se sont donnée ».

À cette date, ventôse an VI, le cercle constitutionnel de la rue de Lille a un rival beaucoup plus actif, celui de la rue du Bac, qui ambitionne de se constituer en Club central du mouvement démocratique. Le bilan de ses travaux qu'il présente le 8 ventôse an VI, se place aussi sous l'égide de la défense de la République constitutionnelle contre la « faction du Prétendant », mais cette République est placée sous l'égide de la Vertu, et non sous celle de la Puissance, différence notable par rapport à Constant. L'essentiel des travaux tourne autour de la préparation des élections, qui doivent commencer à la fin du mois. Centre d'opinion publique certes, mais aussi et surtout centre d'action, comité électoral,

(25) Le Moniteur, ${ }^{\circ}$ 156, 6 ventôse an VI (24 février 1798); article de Lenoir-Laroche, qui était ministre de la police au moment du 18 fructidor. 
dans une logique de proto-formation des partis politiques tout à fait étrangère, voire irréductible à l'univers de Constant, même si ce dernier parviendra à se faire élire à des fonctions locales ${ }^{26}$. Cette évolution des activités du cercle de la rue du Bac provoque d'ailleurs, une semaine plus tard, sa fermeture :

«Le Directoire Exécutif, informé que, sous le nom de Cercle constitutionnel, il se tient, rue du Bac, à Paris, un club dans lequel on a manifestement professé des principes contraires à la Constitution de l'an 3.

Considérant que, s'il importe pour les progrès de la liberté et des lumières, de maintenir les réunions qui, en s'occupant d'objets politiques, ne tendent qu'à fortifier l'esprit public, et à rallier tous les citoyens au gouvernement républicain, il n'est pas moins nécessaire d'arrêter les funestes effets que produisent celles de ces réunions où l'on tendrait au renversement de la constitution, au bouleversement de la République, et où l'on éloignerait les citoyens du gouvernement républicain, en égarant les uns, et en frappant les autres de crainte.

Arrête, en vertu de l'article 37 de la loi du 19 fructidor, que le club qui se rassemble rue du Bac à Paris, sera fermé $»^{27}$.

Et la boucle est bouclée; la législation d'exception adoptée au lendemain du 18 fructidor pour comprimer la droite, se tourne contre la gauche néo-jacobine. Constant n'a certainement pas désavoué cet arrêté, ni ce processus involutif. Le divorce est consommé entre les républicains conservateurs, et les néo-jacobins, qui entament un processus d'autonomie politique, qui va les conduire un an plus tard à formuler un programme de démocratie représentative ${ }^{28}$.

$\mathrm{Au}$ lendemain du 18 fructidor, paraissait un journal significativement intitulé Le Conservateur, dirigé par trois figures éminentes de ce courant républicain conservateur, Daunou, Garat et Marie-Joseph Chénier. Dans l'hiver, était paru un article tout à fait intéressant, signé Garat, qui énonçait des Considérations sur la dictature et les dictateurs. Ces réflexions étaient suscitées par la fermeture du Cercle constitutionnel de la rue de Lille. Il faudrait donc supposer une autodissolution du Cercle, bien avant le transfert de ses activités signalé

(26) Résumé des travaux du Cercle constitutionnel de la rue du Bacq, depuis son organisation jusqu'au 8 ventôse an VI de la République, Paris, BNF, Lb ${ }^{40} 2363$.

(27) Le Moniteur, $\mathrm{n}^{\circ}$ 168, 18 ventôse an VI, p. 673-674.

(28) Bernard GAINOT, 1799, un nouveau jacobinisme? La démocratie représentative, une alternative à Brumaire, Paris, CTHS, 2001. 
par Lenoir-Laroche. Divergences entre ses principaux animateurs? ou signal politique lancé en direction de l'opinion pour indiquer les limites du front républicain, au-delà desquelles le groupe conservateur n'entend pas s'aventurer? L'un des directeurs de ce journal, MarieJoseph Chénier, était ainsi présenté sans indulgence par $\mathrm{M}^{\mathrm{me}}$ de Staël dans les Considérations :

«Chénier était à la fois violent et susceptible de frayeur; plein de préjugés, quoiqu'il fût enthousiaste de la philosophie ; inabordable au raisonnement quand on voulait combattre ses passions, qu'il respectait comme ses dieux pénates. Il se promenait à grands pas dans la chambre, répondait sans avoir écouté, pâlissait, tremblait de colère, lorsqu'un mot qui lui déplaisait frappait tout seul ses oreilles, faute d'avoir la patience d'entendre le reste de la phrase. C'était néanmoins un homme d'esprit et d'imagination, mais tellement dominé par son amour-propre, qu'il s'étonnait de lui-même, au lieu de travailler à se perfectionner ${ }^{29}$.

En 1797, pourtant, on peut supposer que les «passions » évoquées pour Chénier n'étaient pas personnelles, mais partagées par l'ensemble de ce courant « républicain conservateur» qui avait conscience qu'il venait de sauver la République constitutionnelle en violant la Constitution, ainsi que le disait le même Chénier en messidor an III ; rétablir dans le gouvernement la puissance qui risquait de lui échapper en la laissant s'installer dans l'opinion.

Que faut-il retenir de cette intervention de Constant dans ce tournant majeur de la République directoriale? Il s'agit d'abord de l'intervention d'un individu, inséparable de son propre parcours intellectuel. Cette forte empreinte subjective, nous l'avons soulignée en relevant tout ce qui rattache sa prise de position aux écrits de la période antérieure. Certes, on peut y déceler les indices d'une politique de l'intérêt, mais s'arrêter là serait par trop réducteur. C'est aussi un engagement où l'orateur franchit le pas pour se faire, non plus simple publiciste, mais pédagogue de l'opinion publique. Cette dernière est insuffisante pour tracer la marche du gouvernement. Elle doit être relayée par l'esprit public. Constant rejoint, sans équivoque aucune, le camp des républicains conservateurs.

La République directoriale n'a pas cessé d'être une république constitutionnelle, en faisant la part de l'état d'urgence, et de la nécessité de réaffirmer la construction institutionnelle. Le pouvoir n'est plus un 
pouvoir constituant, la république est constituée, c'est la raison principale pour laquelle les hommes d'ordre, qui ont en horreur le radicalisme porteur de guerre civile, doivent s'y rallier. Mais elle n'est pas encore instituée, et ce rappel autorise des entorses provisoires à la norme légale.

Se pose alors la question du centre. Je pense qu'on ne peut parler de position centriste, ni pour Boissy d'Anglas, ni pour Benjamin Constant et les conservateurs, car, implicitement, on ne peut admettre ce positionnement que lorsque la République sera stabilisée. Or, la résurgence de l'état d'exception, après le 13 vendémiaire, après le 18 fructidor, prouve qu'elle ne l'est pas. Elle ne le sera pas davantage, en Brumaire, et au-delà, mais alors, la logique sociale l'emporte de plus en plus sur la logique institutionnelle. Dans les écrits de Constant, la réflexion sur le Gouvernement remplace de plus en plus la réflexion sur la République ; mais c'est la question de formes, même s'il admet dans ses écrits politiques ultérieurs que cette question est loin d'être secondaire.

Je continue donc à penser que l'épithète « conservateur » est une clef de lecture plus pertinente que celle de «centriste». Il faut entendre cette épithète, non pas au sens étroit qu'on lui attribue généralement de défense de l'ordre social, mais comme position transitoire dans un processus de stabilisation institutionnelle sur la longue durée. Il y a des lois qui garantissent les libertés publiques, mais les mœurs sont encore imprégnées par les rapports de dépendance de l'Ancien Régime. La position centriste n'est donc pas de mise car elle suppose un équilibre entre la loi et les mœurs. Or, tant qu'il n'y a pas de mœurs républicaines, établies principalement par l'éducation du peuple, il ne peut y avoir de moyen terme entre les deux instances.

Enfin, rien n'indique sur ces questions, à cette époque, et en dépit de divergences qui tiennent aux jugements sur les personnes, et pas sur les faits, un quelconque désaccord entre l'appréciation portée sur le 18 fructidor par Benjamin Constant, et par $\mathrm{M}^{\mathrm{me}}$ de Staël. La reconstruction opérée par cette dernière dans les Considérations est largement une projection téléologique. Au lendemain du coup d'État du 18 fructidor, l'un et l'autre pouvaient considérer que la République était sauvée, mais que l'essentiel restait à faire.

Bernard GAINOT

Institut d'Histoire de la Révolution française Université Paris1 Panthéon-Sorbonne 69 avenue Danièle Casanova 94200 Ivry-sur-Seine bgainot@yahoo.fr 